\title{
between abnormalities detected by magnetic resonance imaging and knee symptoms in early knee osteoarthritis
}

\author{
Seiya Ota ${ }^{1 凶}$, Eiji Sasaki $^{1}$, Shizuka Sasaki ${ }^{1}$, Daisuke Chiba ${ }^{1}$, Yuka Kimura $^{1}$, Yuji Yamamoto $^{1}$,
} Mika Kumagai ${ }^{2}$, Masataka Ando ${ }^{3}$, Eiichi Tsuda ${ }^{4}$ \& Yasuyuki Ishibashi ${ }^{1}$

We investigated the prevalence of magnetic resonance imaging (MRI) findings and their relationship with knee symptoms in women without radiographic evidence of knee osteoarthritis (KOA). This cross-sectional cohort study included 359 Japanese women without radiographic evidence of KOA (Kellgren-Lawrence grade <2). All participants underwent T2-weighted fat-suppressed MRI of their knees. Structural abnormalities (cartilage damage, bone marrow lesions [BMLs], subchondral cysts, bone attrition, osteophytes, meniscal lesions, and synovitis) were scored according to the wholeorgan MRI score method. Knee symptoms were evaluated using the Knee Injury and Osteoarthritis Outcome Score. Participants were divided into early and non-KOA groups based on early KOA classification criteria. Logistic regression analysis was performed to evaluate the relationship between MRI abnormalities and knee symptoms. Cartilage damage was the most common abnormality (43.5\%). The prevalences of cartilage damage, BMLs, subchondral cysts, bone attrition, meniscal lesions, and synovitis were higher in patients with early KOA than in those without. Synovitis (odds ratio $[O R] 2.254, P=0.002)$ and meniscal lesions $(O R 1.479, P=0.031)$ were positively associated with the presence of early KOA. Synovitis was most strongly associated with knee pain and might be a therapeutic target in patients with early KOA.

Knee osteoarthritis (KOA) is a major public health problem in the middle-aged to elderly population and negatively affects patients' activities of daily living (ADL) and quality of life (QOL) ${ }^{1-3}$. Early diagnosis and treatment of KOA are required to reduce the health care burden ${ }^{4}$ by preventing disease development and progression ${ }^{5-8}$. Early KOA is characterized by the presence of knee symptoms in the absence of definitive radiographic abnormalities (Kellgren-Lawrence [KL] grades $0-1$ ). Adults with KOA are more likely to report more knee symptoms the year before they develop radiographic evidence of $\mathrm{KOA}^{9,10}$. Based on this evidence, patients with early KOA may need to be targeted for therapeutic interventions as early as possible.

Pathologic changes found in osteoarthritic joints include degradation of the articular cartilage, thickening of the subchondral bone, osteophyte formation, varying degrees of synovial inflammation, degeneration of ligaments and menisci, and hypertrophy of the knee joint capsule ${ }^{11}$. Although radiographic evaluation cannot detect minute structural abnormalities, such as cartilage degeneration, meniscal lesions, synovitis, bone marrow lesions (BMLs), and subchondral cysts, magnetic resonance imaging (MRI) may allow the detection of these pathological joint tissue changes. In particular, along with knee symptoms, cartilage damage, meniscal lesions, and BMLs detected by MRI are included in the diagnostic criteria for early $\mathrm{KOA}^{5,12}$.

However, data on the structural abnormalities present in knees without radiographic changes and structural changes associated with knee symptoms are scarce. Therefore, the present study aimed to examine the crosssectional association between MRI lesions and knee symptoms in middle-aged women without radiographic

${ }^{1}$ Department of Orthopaedic Surgery, Hirosaki University Graduate School of Medicine, Hirosaki, Aomori, Japan. ${ }^{2}$ Department of Active Life Promotion, Hirosaki University Graduate School of Medicine, Hirosaki, Aomori, Japan. ${ }^{3}$ Department of Diet and Health Sciences, Hirosaki University Graduate School of Medicine, Hirosaki, Aomori, Japan. 'Department of Rehabilitation Medicine, Hirosaki University Graduate School of Medicine, Hirosaki, Aomori, Japan. ${ }^{\varpi}$ email: otaseiya012@gmail.com 


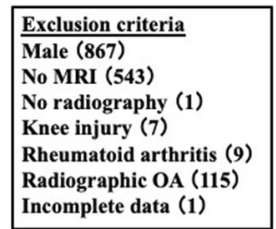

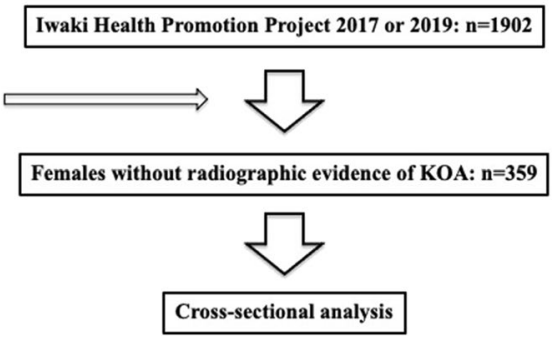

Figure 1. Flow of participants enrollment in Iwaki Health promotion project. Finally, 359 females were enrolled for the statistical analysis.

features of KOA. We hypothesized that the presence of cartilage damage, meniscal lesions, and BMLs are associated with knee symptoms, in accordance with the diagnostic criteria for early KOA.

\section{Methods}

Study design and participants. The participants had previously volunteered for the Iwaki Health Promotion Project, which is a community-based preventive medicine program that aims to improve the average life expectancy by conducting general health checkups and prophylactic interventions, as previously described ${ }^{13-16}$. The ethics committee of the Hirosaki University Graduate School of Medicine approved the study (reference number: 2017-026 and 2019-009), and all subjects provided written informed consent before participation. All methods were performed in accordance with the relevant guidelines and regulations.

In total, 2138 volunteers (876 men and 1262 women) were enrolled in the project in 2017 and 2019. In this study, the analysis of those who participated in both 2017 and $2019(n=236)$ was performed using data from 2017. The participants answered questionnaires about their medical history and current health, lifestyle, and disease-specific information, such as knee symptoms. Height and weight were measured and recorded by the project team staff, and the body mass index (BMI) was calculated. In this study, we focused on women without radiographic abnormalities because the prevalence of KOA is higher in women than in men ${ }^{1,17}$. The exclusion criteria of this study were as follows: men $(n=867)$ and women who lacked knee magnetic resonance (MR) images $(n=543)$, did not undergo radiographic evaluation $(n=1)$, had a history of rheumatoid arthritis $(n=9)$ or knee injury $(n=7)$, had radiographic features of KOA in either knee $(n=115)$, and had incomplete data $(n=1)$. Finally, 359 women without radiographic abnormalities were included in the analysis (Fig. 1).

Radiographic evaluation. The presence of KOA was evaluated using weight-bearing and anterior-posterior radiographs of both knees. The beam was positioned parallel to the floor, with no angle, and aimed at the joint space. KOA severity was classified according to the KL classification ${ }^{18}$. KOA was diagnosed as KL grade $\geq 2$ in the most affected knee. All joints were graded by two orthopedic surgeons (DC and ES), and any discrepancies were resolved by mutual consultation. As aforementioned, only subjects with no radiographic evidence of KOA were included in this analysis. The intra-rater and inter-rater reliabilities, expressed as interclass correlation coefficients (ICC) $(1,1)$ and $(2,1)$ of radiographic evaluation, were 0.952 and 0.931 , respectively.

Knee symptoms. Knee symptoms were scored using a patient-based outcome score, the Knee Injury and Osteoarthritis Outcome Score (KOOS). All participants completed the KOOS questionnaire independently. The KOOS questionnaire is a 42 -item, knee-specific, self-administered questionnaire with five subscales: pain, symptoms, ADL, sports and recreation (sports), and knee-related QOL. All items were scored from 0 to 4 , and the scores were then summed. Next, the raw scores were transformed to a 0-100 scale, with 100 representing the best result and 0 representing the worst ${ }^{1,20}$. Participants were divided into early KOA and non-KOA groups on the basis of their KOOS: a knee was defined as symptomatic when the scores for two of the four KOOS subscales (except KOOS sports) were "positive" (below 85\%), according to the established classification criteria for diagnosing early $\mathrm{KOA}^{6}$. Additionally, participants with joint-line tenderness or crepitation of the knee according to the symptomatic knee were classified as having early KOA. Furthermore, in this study, in order to determine the association between MRI findings and knee pain, a score from 1 to 4 in each KOOS Pain item was defined as "positive," and a score of 0 was defined as "negative".

Knee magnetic resonance imaging. All participants underwent MRI of the right knee using a rapid extremity coil and mobile MR unit (1.5 T; ECHELON RX, Hitachi, Tokyo, Japan) within 1 week of other examinations. The participants were positioned supine with their knees in full extension. Sequences included sagittal and coronal T2-weighted fat saturation fast spin echo (repetition time, $5000 \mathrm{~ms}$; echo time, $80 \mathrm{~ms}$; field-of-view, $16 \mathrm{~cm}$; matrix, $288 \times 288$; and slice thickness, $3 \mathrm{~mm}$ with between-slice gaps of $1.0 \mathrm{~mm}$ ). Evaluation of MRI scans was performed by two independent observers (DC and ES) using the whole-organ MRI score (WORMS) method $^{21}$ in a blinded fashion, with no access to the participants' clinical information. They recorded the presence of abnormalities, such as cartilage damage, BMLs, subchondral cysts, bone attrition, osteophytes, meniscal lesions, and synovitis in each subregion. In the WORMS system, the medial and lateral compartments of 


\begin{tabular}{|c|c|c|c|c|}
\hline & Total sample $(\mathrm{n}=359)$ & Non KOA $(n=305)$ & Early KOA $(n=54)$ & $p$-value \\
\hline Age, years & $51.3 \pm 11.7$ & $50.5 \pm 11.8$ & $55.9 \pm 9.6$ & 0.002 \\
\hline Body mass index, $\mathrm{kg} / \mathrm{m}^{2}$ & $21.9 \pm 3.1$ & $21.8 \pm 3.1$ & $22.5 \pm 3.2$ & 0.101 \\
\hline \multicolumn{5}{|l|}{ KOOS } \\
\hline Symptom & $91.9 \pm 10.8$ & $94.8 \pm 6.5$ & $75.9 \pm 15.4$ & $<0.001$ \\
\hline Pain & $93.4 \pm 12.1$ & $96.8 \pm 6.9$ & $74.0 \pm 16.2$ & $<0.001$ \\
\hline Physical function short form & $95.1 \pm 11.6$ & $98.1 \pm 5.4$ & $77.8 \pm 19.5$ & $<0.001$ \\
\hline Quality of life & $85.7 \pm 18.7$ & $90.8 \pm 13.3$ & $56.9 \pm 18.7$ & $<0.001$ \\
\hline Symptomatic knee, \% & $69(19.2 \%)$ & $15(4.9 \%)$ & $54(100.0 \%)$ & $<0.001$ \\
\hline
\end{tabular}

Table 1. The demographic data of study participants. Values are means \pm standard deviations of age, body mass index, and KOOS. In regards to symptomatic knee, values are represented by prevalence (percentage of the whole population). Differences among the two groups were compared by Mann-Whitney U test and chi-square test. A $p$-value indicates the significance of difference between participants with and without knee symptoms. KOOS Knee injury and Outcome Score.

the tibia and femur are divided into three subregions (anterior, central, and posterior), and the tibia has one additional subregion, representing the area beneath the tibial spine. The patella is divided into medial and lateral subregions. Cartilage damage was scored in each of the 14 articular surfaces (excluding region S) from 0 (normal thickness and signal) to 6 (diffuse $[\geq 75 \%$ of the region] full-thickness loss). BMLs were each scored as integers from 0 to 3 , where $0=$ normal; $1=$ mild, $<25 \%$ of the region; $2=$ moderate, $25-50 \%$ of the region; and $3=$ severe, $>50 \%$ of the region. Subchondral cysts were graded in each region from 0 to 3 based on the extent of regional involvement, as for BMLs. Bone attrition was defined as flattening or depression of the articular surfaces and was graded from 0 to 3 based on the subjective degree of deviation from the normal contour: $0=$ normal, 1 =mild, 2 =moderate, and 3 =severe. Osteophytes along 14 different margins of the knee, the anterior, central weight-bearing, and posterior margins of the femoral condyles and tibial plateaus, and the medial and lateral margins of the patella were graded from 0 to 7 using the following scale: $0=$ none; $1=$ equivocal; $2=$ small; $3=$ small-moderate; $4=$ moderate; $5=$ moderate-large; $6=$ large; and $7=$ very large. Meniscal lesions were graded separately from 0 to 4 : $0=$ intact; $1=$ minor radial tear or parrot-beak tear; $2=$ non-displaced tear or prior surgical repair; 3 = displaced tear or partial resection; and $4=$ complete maceration/destruction or complete resection. Since joint effusion and synovial thickening cannot be distinguished from each other, synovitis was graded collectively from 0 to 3 in terms of the estimated maximal distention of the synovial cavity: $0=$ normal; $1=$ less than $33 \%$ of maximum potential distention; $2=33-66 \%$ of maximum potential distention; and $3=$ more than $66 \%$ of maximum potential distention. For cartilage damage, BMLs, subchondral cysts, bone attrition, and osteophytes, we computed the number of subregions with damage as the number of subregions with a score of $>0$. For meniscal lesions, we computed the maximum meniscal damage grade for the entire knee. The intra-rater and interrater reliabilities, expressed as ICC $(1,1)$ and $(2,1)$ of scoring MRI, were 0.929 and 0.921 , respectively.

Statistical analysis. The mean values of continuous variables (age, BMI, and KOOS) were compared using the Mann-Whitney U test. The chi-square test was used to compare the prevalence of MRI lesions between the non-KOA and early KOA groups. To evaluate the relationship between knee symptoms and MRI lesions, we performed crude and adjusted logistic regression analyses, with the presence of knee symptoms as an independent variable, and age, BMI, and the total score of each MRI lesion as dependent variables. Furthermore, to evaluate the relationship between knee pain and MRI lesions, we performed multiple logistic regression analyses with the presence of knee pain as an independent variable, and age, BMI, and each MRI lesion as dependent variables. Data analysis was conducted using SPSS (version 24.0; IBM Corp., Armonk, NY, USA) in a cross-sectional manner. Statistical significance was set at $P<0.05$.

\section{Results}

Participants' demographics. The mean age of participants was $51.3 \pm 11.7$ years, mean BMI was $21.9 \pm 3.1 \mathrm{~kg} / \mathrm{m}^{2}$. Sixty-nine (19.2\%) participants had symptomatic knees, and $54(15.0 \%)$ met the diagnostic criteria for early KOA (Table 1). The prevalence of early KOA increased with age, exceeding $20 \%$ in participants aged 60 years and older (Fig. 2). The prevalences of cartilage damage, BMLs, subchondral cysts, bone attrition, osteophytes, meniscal lesions, and synovitis were $43.5 \%, 28.7 \%, 9.2 \%, 6.4 \%, 42.6 \%, 12.3 \%$, and $25.9 \%$, respectively. The severities of cartilage damage $(P=0.025)$, BMLs $(P=0.026)$, subchondral cysts $(P=0.048)$, bone attrition $(P=0.012)$, meniscal lesions $(P<0.001)$, and synovitis $(P<0.001)$ were significantly higher among participants with early KOA than among those without KOA (Tables 2, 3).

Association between early knee osteoarthritis and magnetic resonance imaging lesions. According to the logistic regression analysis, cartilage damage (odds ratio [OR] 1.463, 95\% confidence interval [CI] 1.085, 1.974), BMLs (OR 1.499, 95\% CI 1.098, 2.047), subchondral cysts (OR 2.201, 95\% CI $1.112,4.355$ ), bone attrition (OR 3.362, 95\% CI 1.350, 8.376), meniscal lesions (OR 1.836, 95\% CI 1.332, 2.530), and synovitis (OR 2.779, 95\% CI 1.736, 4.449) were significantly associated with the presence of early KOA. 


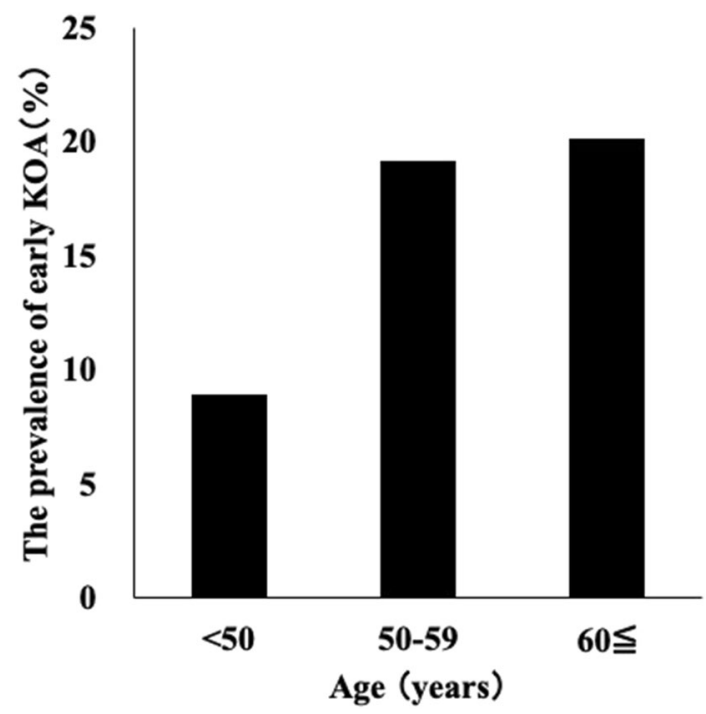

Figure 2. The prevalence of early knee osteoarthritis (KOA) classified by age.

\begin{tabular}{|c|c|c|c|c|}
\hline & Total sample $(n=359)$ & Non KOA $(n=305)$ & Early KOA $(n=54)$ & $p$-value \\
\hline \multicolumn{5}{|c|}{ No. of subregions with cartilage score $>0$ across entire knee } \\
\hline $0-1$ & $312(86.9)$ & $271(88.9)$ & $41(75.9)$ & \multirow{3}{*}{0.025} \\
\hline $2-4$ & $46(12.8)$ & $33(10.8)$ & $13(24.1)$ & \\
\hline $5+$ & $1(0.3)$ & $1(0.3)$ & $0(0.0)$ & \\
\hline \multicolumn{5}{|c|}{ No. of subregions affected by any BML } \\
\hline 0 & $256(71.3)$ & $226(74.1)$ & $30(55.6)$ & \multirow{6}{*}{0.026} \\
\hline 1 & $75(20.9)$ & $58(19.0)$ & $17(31.5)$ & \\
\hline 2 & $20(5.6)$ & $16(5.2)$ & $4(7.4)$ & \\
\hline 3 & $4(1.1$ & $3(1.0)$ & $1(1.9)$ & \\
\hline 4 & $3(0.8)$ & $1(0.3)$ & $2(3.7)$ & \\
\hline $5+$ & $1(0.3)$ & $1(0.3)$ & $0(0.0)$ & \\
\hline \multicolumn{5}{|c|}{ No. of subregions affected by any subchondral cyst } \\
\hline 0 & $326(90.8)$ & $281(92.1)$ & $45(83.3)$ & \multirow{3}{*}{0.048} \\
\hline 1 & $29(8.1)$ & $22(7.2)$ & $7(13.0)$ & \\
\hline 2 & $4(1.1)$ & $2(0.7)$ & $2(3.7)$ & \\
\hline
\end{tabular}

Table 2. The distribution of MRI lesions of study participants. The values represent the number (percentage of the whole population) of MRI lesions of all participants, participants with early KOA, and those without KOA. Differences among the two groups were compared by chi-square test. A $p$-value indicates the significance of difference between participants with and without early KOA.

Furthermore, an adjusted regression model showed that meniscal lesions (OR 1.479, 95\% CI 1.036, 2.110) and synovitis (OR $2.254,95 \%$ CI 1.356, 3.747) were positively correlated with the presence of early KOA (Table 4).

Association between knee pain and magnetic resonance imaging lesions. In terms of the association between MRI lesions and knee pain with certain motions, those with early KOA suffered from pain when going up or down stairs, bending the knee, or performing sitting or lying down motions (Table 5). Additionally, regression analysis showed that synovitis and meniscal lesions were positively associated with almost all KOOS pain items. On the other hand, cartilage was not significantly associated with any KOOS pain item, except for pain with going up or down stairs. BMLs tended to be associated with pain when straightening the knee $(P=0.067$, OR $1.387,95 \%$ CI $0.977,1.968)$, bending the knee $(P=0.066$, OR $1.340,95 \%$ CI $0.981,1.830)$, and walking on a flat surface $(P=0.039$, OR $1.453,95 \%$ CI 1.019, 2.073) (Table 6).

\section{Discussion}

The most important findings of the present study were that MRI findings in early KOA diagnosed by the newly established criteria were proven, and imaging evidence of various types of knee pain was demonstrated. Although MRI evaluation showed that cartilage damage (44\%) was the most common finding in women without 


\begin{tabular}{|c|c|c|c|c|}
\hline & Total sample $(\mathrm{n}=359)$ & Non KOA $(n=305)$ & Early KOA $(n=54)$ & $p$-value \\
\hline \multicolumn{5}{|c|}{ No. of subregions affected by any attrition } \\
\hline 0 & $336(93.6)$ & $290(95.1)$ & $46(85.2)$ & \multirow{2}{*}{0.012} \\
\hline 1 & $23(6.4)$ & $15(4.9)$ & $8(14.8)$ & \\
\hline \multicolumn{5}{|c|}{ No. of locations affected by any osteophyte } \\
\hline $0-2$ & $349(97.2)$ & $298(97.7)$ & $51(94.4)$ & \multirow{2}{*}{0.178} \\
\hline $3+$ & $10(2.8)$ & $7(2.3)$ & $3(5.6)$ & \\
\hline \multicolumn{5}{|c|}{ Maximum grade of meniscal lesion across all locations } \\
\hline 0 & $315(87.7)$ & $276(90.5)$ & $39(72.2)$ & \multirow{3}{*}{$<0.001$} \\
\hline $1-2$ & $33(9.2)$ & $24(7.9)$ & $9(16.7)$ & \\
\hline $3+$ & $11(3.1)$ & $5(1.6)$ & $6(11.1)$ & \\
\hline \multicolumn{5}{|c|}{ Synovitis } \\
\hline $0-1$ & $350(97.5)$ & $303(99.3)$ & $47(87.0)$ & \multirow{2}{*}{$<0.001$} \\
\hline $2-3$ & $9(2.5)$ & $2(0.7)$ & $7(13.0)$ & \\
\hline
\end{tabular}

Table 3. The distribution of MRI lesions of study participants. The values represent the number (percentage of the whole population) of MRI lesions of all participants, participants with early KOA, and those without KOA. Differences among the two groups were compared by chi-square test. A $p$-value indicates the significance of difference between participants with and without early KOA.

\begin{tabular}{|l|c|l|l|l|l|l|}
\hline \multirow{2}{*}{ Independent variables } & \multicolumn{4}{l}{ Non-adjusted } & \multicolumn{2}{l|}{ Adjusted } \\
\cline { 2 - 7 } & $\boldsymbol{p}$-value & Odds ratio & $\mathbf{9 5 \%}$ CI & $\boldsymbol{p}$-value & Odds ratio & $\mathbf{9 5 \% ~ C I ~}$ \\
\hline Cartilage & 0.013 & 1.463 & $1.082-1.974$ & 0.983 & 0.996 & $0.693-1.431$ \\
\hline Bone marrow lesions & 0.011 & 1.499 & $1.098-2.047$ & 0.363 & 1.179 & $0.826-1.684$ \\
\hline Subchondral cysts & 0.024 & 2.201 & $1.112-4.355$ & 0.155 & 1.711 & $0.816-3.591$ \\
\hline Attrition & 0.009 & 3.362 & $1.350-8.376$ & 0.650 & 1.292 & $0.427-3.910$ \\
\hline Osteophytes & 0.092 & 1.297 & $0.958-1.757$ & & & \\
\hline Meniscal lesions & $<0.001$ & 1.836 & $1.332-2.530$ & 0.031 & 1.479 & $1.036-2.110$ \\
\hline Synovitis & $<0.001$ & 2.779 & $1.736-4.449$ & 0.002 & 2.254 & $1.356-3.747$ \\
\hline
\end{tabular}

Table 4. MRI lesion related to the presence of early knee osteoarthritis. Logistic regression analysis was performed on knees with Kellgren-Lawrence grade 0 or 1 in radiographs. In non-adjusted model, dependent variables was the presence of early KOA; the independent variables were each MRI lesion. In adjusted model, dependent variables was the presence of early KOA; the independent variables were age, BMI, MRI lesions except osteophytes.

\begin{tabular}{|l|l|l|l|l|}
\hline & Total sample $(\mathbf{n}=\mathbf{3 5 9})$ & Non KOA $(\mathbf{n}=\mathbf{3 0 5})$ & Early KOA $(\mathbf{n}=\mathbf{5 4})$ & $\boldsymbol{p}$-value \\
\hline How often do you experience knee pain? & $118(32.9)$ & $67(22.0)$ & $51(94.4)$ & $<0.001$ \\
\hline Twisting/pivoting on your knee & $69(19.2)$ & $34(11.1)$ & $35(64.8)$ & $<0.001$ \\
\hline Straightening knee fully & $43(12.0)$ & $16(5.2)$ & $27(50.0)$ & $<0.001$ \\
\hline Bending knee fully & $68(18.9)$ & $27(8.9)$ & $41(75.9)$ & $<0.001$ \\
\hline Walking on flat surface & $38(10.6)$ & $16(5.2)$ & $22(40.7)$ & $<0.001$ \\
\hline Going up or down stairs & $87(24.2)$ & $44(14.4)$ & $43(79.6)$ & $<0.001$ \\
\hline At night while in bed & $25(7.0)$ & $11(3.6)$ & $14(25.9)$ & $<0.001$ \\
\hline Sitting or lying & $73(20.3)$ & $34(11.1)$ & $39(72.2)$ & $<0.001$ \\
\hline Standing upright & $38(10.6)$ & $18(5.9)$ & $20(37.0)$ & $<0.001$ \\
\hline
\end{tabular}

Table 5. The prevalence of each knee pains of study participants. The values represent the prevalence of each knee pains of all participants, participants with early KOA, and those without KOA. Data are based on the number of participants (percentage of the whole population) for each knee pains. Differences among the two groups were compared by chi-square test. A $p$-value indicates the significance of difference between participants with and without early KOA. 


\begin{tabular}{|c|c|c|c|c|c|c|c|}
\hline & Cartilage & Bone marrow lesions & Subchondral cysts & Attrition & Ostcephytes & Meniscal lesions & Synovitis \\
\hline $\begin{array}{l}\text { How often do you experience } \\
\text { knee pain? }\end{array}$ & $1.11(0.85-1.45)$ & $1.17(0.88-1.56)$ & $1.05(0.55-2.00)$ & $2.51(1.05-5.99)$ & $1.07(0.82-1.39)$ & $1.73(1.22-2.44)$ & $2.04(1.34-3.11)$ \\
\hline Twisting/pivoting on your knee & $1.09(0.80-1.48)$ & $1.26(0.92-1.72$ & $1.17(0.57-2.41)$ & $3.24(1.34-7.84)$ & $1.32(0.99-1.76)$ & $1.51(1.11-2.06)$ & $1.70(1.09-2.65)$ \\
\hline Straightening knee fully & $0.94(0.64-1.38)$ & $1.39(0.98-1.97)$ & $0.97(0.39-2.39)$ & $1.43(0.45-4.49)$ & $1.16(0.82-1.64)$ & 1.41 (1.01-1.98) & $1.82(1.10-2.98)$ \\
\hline Bending knee fully & $1.16(0.86-1.57)$ & $1.34(0.98-1.83)$ & $1.35(0.67-2.72)$ & $2.10(0.84-5.26)$ & $1.27(0.95-1.70)$ & $1.59(1.16-2.17)$ & $1.65(1.06-2.58)$ \\
\hline Walking on flat surface & $0.97(0.65-1.45)$ & $1.45(1.02-2.07)$ & $1.10(0.45-2.72)$ & $2.22(0.76-6.51)$ & $1.11(0.77-1.60)$ & $1.23(0.84-1.79)$ & $2.01(1.20-3.35)$ \\
\hline Going up or down stairs & $1.35(1.02-1.79)$ & $1.26(0.93-1.70)$ & $1.72(0.90-3.29)$ & $3.51(1.46-8.43)$ & $1.49(1.13-1.96)$ & $1.78(1.27-2.48)$ & $2.08(1.35-3.20)$ \\
\hline At night while in bed & $1.18(0.76-1.83)$ & $1.33(0.85-2.07)$ & $0.64(0.16-2.52)$ & $2.11(0.56-7.91)$ & $1.72(1.17-2.51)$ & $1.59(1.10-2.29)$ & $1.51(0.81-2.80)$ \\
\hline Sitting or lying & $0.97(0.72-1.32)$ & $1.20(0.88-1.63)$ & $1.13(0.55-2.31)$ & $1.71(0.68-4.34)$ & $1.11(0.83-1.50)$ & $1.15(0.83-1.59)$ & $1.97(1.26-3.07)$ \\
\hline Standing upright & $0.79(0.51-1.24)$ & $1.16(0.78-1.72)$ & $1.11(0.45-2.74)$ & $1.68(0.53-5.32)$ & $1.28(0.90-1.82)$ & $1.51(1.08-2.11)$ & $1.86(1.11-3.12)$ \\
\hline
\end{tabular}

Table 6. The association between MRI lesions and knee pains with certain activities. Logistic regression analysis was performed on knees with Kellgren-Lawrence grade 0 or 1 in radiographs. Dependent variables was the presence of each knee pains; the independent variables were age, BMI, and each MRI lesion. Bold denotes statistical significance at $P<0.05$.

radiographic knee abnormalities, there was no significant association with the presence of early KOA. The prevalence of BMLs (29\%), subchondral cysts (9\%), bone attrition (6\%), meniscal lesions (12\%), and synovitis (26\%) were higher in patients with early KOA than in those without. Meniscal lesions and synovitis were positively associated with the presence of early KOA and key factors for various types of knee pain. These results suggest that meniscal lesions and synovitis have the greatest impact on the symptoms of patients with early KOA, thus proving our hypothesis; these should be detected and appropriate intervention should be applied to prevent disease progression.

Few studies have investigated the prevalence of abnormalities detected by MRI, specifically in a population without radiographic KOA. Guermazi et al. reported that osteophytes were the most common abnormality (74\%), followed by cartilage damage (69\%), BMLs (52\%), synovitis (37\%), and bone attrition (32\%) in participants without radiographic evidence of $\mathrm{KOA}^{22}$. Javaid et al. found that osteophytes (99\%), cartilage damage (70\%), and BMLs (59\%) were common in participants without knee symptoms and radiographic $\mathrm{KOA}^{23}$. In the current study, the same tendency was observed, that is, many participants had cartilage damage, osteophytes, and BMLs. However, the prevalence of each abnormality was lower than that reported in previous studies. This may be because the participants in our study were younger, had a lower BMI, and might have a lower risk of KOA than those in previous studies.

Many previous studies have reported an association between knee effusion and knee symptoms. Torres et al. noted that synovitis or effusion detected on MRI correlated best with knee pain measured on a visual analog scale $^{24}$. Chiba et al. investigated suprapatellar effusion using quantitative ultrasonography and concluded that knee effusion was associated with symptoms as evaluated by $\mathrm{KOOS}^{13}$. Moreover, several studies have shown that knee effusion is associated with the KOA disease stage and progression ${ }^{14,25,26}$. Although there have been few studies of effusion in early KOA, Harkey et al. suggested that effusion precedes the onset of accelerated KOA and may be a prognostic biomarker ${ }^{27}$. In the present study, synovitis was the most strongly associated with knee symptoms and was considered to be the most important clinical finding indicating the need for intervention, which is in agreement with findings of previous reports.

Meniscal lesions and BMLs have also been associated with knee symptoms and disease progression in previous studies $^{28-31}$. In addition, there have been reports that meniscal lesions, BMLs, and synovitis are associated with each other ${ }^{32-34}$. However, van Oudenaarde et al. suggested that the discriminative power of single MRI features is insufficient to be useful as a predictor of $\mathrm{KOA}^{35}$. In our study, although meniscal lesions, together with cartilage damage, BMLs, subchondral cysts, bone attrition, and synovitis, were significantly associated with the presence of early KOA, the adjusted regression model showed that this association was attenuated after adjustment for age and BMI. In the early KOA population, synovitis contributes more to knee symptoms than meniscal lesions.

BMLs reflect bony damage, which is associated with knee symptoms in patients with KOA and bone metabolism, such as bone absorption. In recent studies, the presence of BMLs has been associated with knee pain and predicted cartilage loss in patients with established $\mathrm{KOA}^{36-38}$. Antiresorptive drugs (e.g., bisphosphonate) were found to reduce the size of BMLs and the risk of total knee arthroplasty in patients with KOA ${ }^{39,40}$. In early KOA, lower bone mineral density and higher levels of some bone markers were associated with the presence of BMLs ${ }^{15}$. In the present study, BMLs tended to be associated with pain in relatively loaded motions, such as bending and straightening the knees and walking. BMLs may be induced by bone fragility, resulting in early subchondral changes, such as microcracking before radiologic osteoarthritic findings become definitive and cause pain during loading.

In many previous studies, although cartilage damage was associated with bone marrow lesions ${ }^{41,42}$, meniscal damage $^{43}$, and synovitis or effusion ${ }^{44}$, cartilage damage was not always associated with the severity of knee pain ${ }^{22}$. Since articular cartilage is not innervated, the association between cartilage and pain severity may be due to other structural abnormalities associated with $\mathrm{KOA}^{45}$. In this study, cartilage damage was the most common finding, but it was not significantly associated with pain. This result suggested that minor cartilage damage detected by MRI was not associated with knee symptoms in accordance with the established KOA. 
This study had several limitations. First, all participants in this study were women. Although the prevalence of KOA is higher in women than in men, men may be more likely to experience knee trauma, which may affect the prevalence of cartilage damage, BMLs, and meniscal lesions. Second, laboratory data, including inflammation and cartilage/bone metabolic markers, were not evaluated. Serum hyaluronic acid, a marker of synovitis, has been assessed in many previous studies ${ }^{14,46}$. Third, the data of synovitis were obtained using non-contrast images, which are less sensitive in distinguishing effusion from underlying synovial tissue than contrast images. Because of the possible complications and increased cost, we did not use contrast-enhanced MRI. Fourth, the findings of this study were based on cross-sectional data; therefore, future longitudinal studies are needed to investigate whether patients with knee effusion are likely to develop KOA. In addition, because of some findings, such as repeated remission and exacerbation of BMLs, caution may be required when interpreting the results of this study.

\section{Conclusions}

Structural abnormalities detected by MRI, such as cartilage damage, osteophytes, and BMLs, were commonly present in the knees of middle-aged Japanese women with no radiographic evidence of KOA. Among MRI findings, synovitis had the most marked effect on knee pain and might be a therapeutic target in patients with early KOA.

\section{Data availability}

The data to reproduce the analyses are available at https://drive.google.com/drive/folders/1Ch7_IH8cDUdYii tqIABBsAfHosZbUsMm? usp=sharing.

Received: 17 March 2021; Accepted: 6 July 2021

Published online: 26 July 2021

\section{References}

1. Yoshimura, N. et al. Prevalence of knee osteoarthritis, lumbar spondylosis, and osteoporosis in Japanese men and women: The research on osteoarthritis/osteoporosis against disability study. J. Bone Miner. Metab. 27, 620-628. https://doi.org/10.1007/s00774009-0080-8 (2009).

2. Cross, M. et al. The global burden of hip and knee osteoarthritis: Estimates from the global burden of disease 2010 study. Ann. Rheum. Dis. 73, 1323-1330. https://doi.org/10.1136/annrheumdis-2013-204763 (2014).

3. Kluzek, S. et al. Painful knee but not hand osteoarthritis is an independent predictor of mortality over 23 years follow-up of a population-based cohort of middle-aged women. Ann. Rheum. Dis. 75, 1749-1756. https://doi.org/10.1136/annrheumdis-2015208056 (2016).

4. Losina, E. et al. Pharmacologic regimens for knee osteoarthritis prevention: Can they be cost-effective?. Osteoarthr. Cartil. 22, 415-430. https://doi.org/10.1016/j.joca.2014.01.005 (2014).

5. Madry, H. et al. Early osteoarthritis of the knee. Knee Surg. Sports Traumatol. Arthrosc. 24, 1753-1762. https://doi.org/10.1007/ s00167-016-4068-3 (2016).

6. Luyten, F. P. et al. Toward classification criteria for early osteoarthritis of the knee. Semin. Arthritis Rheum. 47, 457-463. https:// doi.org/10.1016/j.semarthrit.2017.08.006 (2018).

7. Emery, C. A. et al. Establishing outcome measures in early knee osteoarthritis. Nat. Rev. Rheumatol. 15, 438-448. https://doi.org/ 10.1038/s41584-019-0237-3 (2019).

8. Sasaki, E. et al. Early knee osteoarthritis prevalence is highest among middle-aged adult females with obesity based on new set of diagnostic criteria from a large sample cohort study in the Japanese general population. Knee Surg. Sports Traumatol. Arthrosc. 28, 984-994. https://doi.org/10.1007/s00167-019-05614-z (2020).

9. Case, R., Thomas, E., Clarke, E. \& Peat, G. Prodromal symptoms in knee osteoarthritis: A nested case-control study using data from the osteoarthritis Initiative. Osteoarthr. Cartil. 23, 1083-1089. https://doi.org/10.1016/j.joca.2014.12.026 (2015).

10. Driban, J. B. et al. Individuals with incident accelerated knee osteoarthritis have greater pain than those with common knee osteoarthritis progression: Data from the osteoarthritis Initiative. Clin. Rheumatol. 35, 1565-1571. https://doi.org/10.1007/s10067015-3128-2 (2016).

11. Loeser, R. F., Goldring, S. R., Scanzello, C. R. \& Goldring, M. B. Osteoarthritis: A disease of the joint as an organ. Arthritis Rheum. 64, 1697-1707. https://doi.org/10.1002/art.34453 (2012).

12. Luyten, F. P., Denti, M., Filardo, G., Kon, E. \& Engebretsen, L. Definition and classification of early osteoarthritis of the knee. Knee Surg. Sports Traumatol. Arthrosc. 20, 401-406. https://doi.org/10.1007/s00167-011-1743-2 (2012).

13. Chiba, D. et al. Evaluation of a quantitative measurement of suprapatellar effusion by ultrasonography and its association with symptoms of radiographic knee osteoarthritis: A cross-sectional observational study. Arthritis Res. Ther. 18, 181. https://doi.org/ 10.1186/s13075-016-1078-y (2016).

14. Sasaki, E. et al. Serum hyaluronic acid concentration predicts the progression of joint space narrowing in normal knees and established knee osteoarthritis: A five-year prospective cohort study. Arthritis Res. Ther. 17, 283. https://doi.org/10.1186/s13075015-0793-0 (2015)

15. Ota, S. et al. Symptomatic bone marrow lesions induced by reduced bone mineral density in middle-aged women: A cross-sectional Japanese population study. Arthritis Res. Ther. 21, 113. https://doi.org/10.1186/s13075-019-1900-4 (2019).

16. Inoue, R. et al. Knee osteoarthritis, knee joint pain and aging in relation to increasing serum hyaluronan level in the Japanese population. Osteoarthr. Cartil. 19, 51-57. https://doi.org/10.1016/j.joca.2010.10.021 (2011).

17. Felson, D. T. et al. The prevalence of knee osteoarthritis in the elderly. The Framingham osteoarthritis Study. Arthritis Rheum. 30, 914-918. https://doi.org/10.1002/art.1780300811 (1987) (Pubmed:3632732).

18. Kellgren, J. H. \& Lawrence, J. S. Radiological assessment of osteo-arthrosis. Ann. Rheum. Dis. 16, 494-502. https://doi.org/10. 1136/ard.16.4.494 (1957).

19. Roos, E. M., Roos, H. P., Lohmander, L. S., Ekdahl, C. \& Beynnon, B. D. Knee Injury and Osteoarthritis Outcome Score (KOOS): Development of a self-administered outcome measure. J. Orthop. Sports Phys. Ther. 28, 88-96. https://doi.org/10.2519/jospt.1998. 28.2.88 (1998)

20. Nakamura, N. et al. Cross-cultural adaptation and validation of the Japanese Knee Injury and Osteoarthritis Outcome Score (KOOS). J. Orthop. Sci. 16, 516-523. https://doi.org/10.1007/s00776-011-0112-9 (2011).

21. Peterfy, C. G. et al. Whole-organ magnetic resonance imaging score (WORMS) of the knee in osteoarthritis. Osteoarthr. Cartil. 12, 177-190. https://doi.org/10.1016/j.joca.2003.11.003 (2004).

22. Guermazi, A. et al. Prevalence of abnormalities in knees detected by MRI in adults without knee osteoarthritis: Population based observational study (Framingham osteoarthritis Study). BMJ 345, e5339. https://doi.org/10.1136/bmj.e5339 (2012). 
23. Javaid, M. K. et al. Pre-radiographic MRI findings are associated with onset of knee symptoms: The most study. Osteoarthr. Cartil. 18, 323-328. https://doi.org/10.1016/j.joca.2009.11.002 (2010).

24. Torres, L. et al. The relationship between specific tissue lesions and pain severity in persons with knee osteoarthritis. Osteoarthr. Cartil. 14, 1033-1040. https://doi.org/10.1016/j.joca.2006.03.015 (2006).

25. Ayral, X., Pickering, E. H., Woodworth, T. G., Mackillop, N. \& Dougados, M. Synovitis: A potential predictive factor of structural progression of medial tibiofemoral knee osteoarthritis: Results of a 1 year longitudinal arthroscopic study in 422 patients. Osteoarthr. Cartil. 13, 361-367. https://doi.org/10.1016/j.joca.2005.01.005 (2005).

26. Krasnokutsky, S. et al. Quantitative magnetic resonance imaging evidence of synovial proliferation is associated with radiographic severity of knee osteoarthritis. Arthritis Rheumatol. 63, 2983-2991 (2001).

27. Harkey, M. S. et al. Early pre-radiographic structural pathology precedes the onset of accelerated knee osteoarthritis. BMC Musculoskelet. Disord. 20, 241. https://doi.org/10.1186/s12891-019-2624-y (2019).

28. Felson, D. T. et al. The association of bone marrow lesions with pain in knee osteoarthritis. Ann. Intern. Med. 134, 541-549. https:// doi.org/10.7326/0003-4819-134-7-200104030-00007 (2001).

29. Dore, D. et al. Bone marrow lesions predict site-specific cartilage defect development and volume loss: A prospective study in older adults. Arthritis Res. Ther. 12, R222. https://doi.org/10.1186/ar3209 (2010).

30. Kim, H. A. et al. The association between meniscal and cruciate ligament damage and knee pain in community residents. Osteoarthr. Cartil. 19, 1422-1428. https://doi.org/10.1016/j.joca.2011.09.002 (2011).

31. Chiba, D. et al. Meniscal extrusion seen on ultrasonography affects the development of radiographic knee osteoarthritis: A 3-year prospective cohort study. Clin. Rheumatol. 36, 2557-2564. https://doi.org/10.1007/s10067-017-3803-6 (2017).

32. Lo, G. H. et al. Strong association of MRI meniscal derangement and bone marrow lesions in knee osteoarthritis: Data from the osteoarthritis initiative. Osteoarthr. Cartil. 17, 743-747. https://doi.org/10.1016/j.joca.2008.11.014 (2009).

33. Roemer, F. W. et al. The association between meniscal damage of the posterior horns and localized posterior synovitis detected on T1-weighted contrast-enhanced MRI: The MOST study. Semin. Arthritis Rheum. 42, 573-581. https://doi.org/10.1016/j.semar thrit.2012.10.005 (2013).

34. Wang, X. et al. Association between MRI-detected knee joint regional effusion-synovitis and structural changes in older adults: A cohort study. Ann. Rheum. Dis. 75, 519-525. https://doi.org/10.1136/annrheumdis-2014-206676 (2016).

35. van Oudenaarde, K. et al. Predictive value of MRI features for development of radiographic osteoarthritis in a cohort of participants with pre-radiographic knee osteoarthritis-the CHECK study. Rheumatology (Oxford) 56, 113-120. https://doi.org/10.1093/rheum atology/kew368 (2017).

36. Kothari, A. et al. Within-subregion relationship between bone marrow lesions and subsequent cartilage loss in knee osteoarthritis. Arthritis Care Res. 62, 198-203. https://doi.org/10.1002/acr.20068 (2010).

37. Driban, J. B. et al. Quantitative bone marrow lesion size in osteoarthritic knees correlates with cartilage damage and predicts longitudinal cartilage loss. BMC Musculoskelet. Disord. 12, 217. https://doi.org/10.1186/1471-2474-12-217 (2011).

38. Driban, J. B. et al. Evaluation of bone marrow lesion volume as a knee osteoarthritis biomarker: Longitudinal relationships with pain and structural changes: Data from the osteoarthritis Initiative. Arthritis Res. Ther. 15, R112. https://doi.org/10.1186/ar4292 (2013).

39. Laslett, L. L. et al. Zoledronic acid reduces knee pain and bone marrow lesions over 1 year: A randomised controlled trial. Ann. Rheum. Dis. 71, 1322-1328. https://doi.org/10.1136/annrheumdis-2011-200970 (2012).

40. Fu, S. H., Wang, C. Y., Yang, R. S., Wu, F. L. \& Hsiao, F. Y. Bisphosphonate use and the risk of undergoing total knee arthroplasty in osteoporotic patients with osteoarthritis: A nationwide cohort study in Taiwan. J. Bone Joint Surg. Am. 99, 938-946. https://doi. org/10.2106/JBJS.16.00385 (2017).

41. Roemer, F. W. et al. Change in MRI-detected subchondral bone marrow lesions is associated with cartilage loss: The MOST Study: A longitudinal multicentre study of knee osteoarthritis. Ann. Rheum. Dis. 68, 1461-1465. https://doi.org/10.1136/ard.2008.096834 (2009).

42. Antony, B. et al. Correlates of knee bone marrow lesions in younger adults. Arthritis Res. Ther. 18, 31. https://doi.org/10.1186/ s13075-016-0938-9 (2016).

43. Hunter, D. J. et al. The association of meniscal pathologic changes with cartilage loss in symptomatic knee osteoarthritis. Arthritis Rheum. 54, 795-801. https://doi.org/10.1002/art.21724 (2006).

44. Roemer, F. W. et al. Tibiofemoral joint osteoarthritis: Risk factors for MR-depicted fast cartilage loss over a 30-month period in the multicenter osteoarthritis study. Radiology 252, 772-780. https://doi.org/10.1148/radiol.2523082197 (2009).

45. Hunter, D. J. et al. Evolution of semi-quantitative whole joint assessment of knee OA: MOAKS (MRI Osteoarthritis Knee Score). Osteoarthr. Cartil. 19, 990-1002. https://doi.org/10.1016/j.joca.2011.05.004 (2011).

46. Ishijima, M. et al. Relationships between biomarkers of cartilage, bone, synovial metabolism and knee pain provide insights into the origins of pain in early knee osteoarthritis. Arthritis Res. Ther. 13, R22. https://doi.org/10.1186/ar3246 (2011).

\section{Acknowledgements}

We are extremely grateful to all participants in the Iwaki Health Promotion Project and to the staff of our department, who conducted interviews and collected the data. We would like to thank Editage (www.editage.jp) for English language editing.

\section{Author contributions}

S.O., E.S., and D.C. contributed to the conception and design of the study. S.O., M.K., and M.A. contributed to data acquisition. S.O., E.S., S.S., D.C., Y.K., and Y.Y. contributed to the analysis and interpretation of the data. SO and ES drafted the manuscript. Y.Y., E.T., and Y.I. critically revised the manuscript for important intellectual content. S.O., E.S., S.S., D.C., Y.K., Y.Y., M.K., M.A., E.T., and Y.I. approved the final version of the manuscript for publication. Y.I. is the guarantor. The corresponding author attests that all listed authors meet the authorship criteria and that no other authors meeting the criteria have been omitted.

\section{Funding}

This study was supported in part by a Grant-in-Aid from the Japanese Society for the Promotion of Science (Nos. 21500676, 18K16606, and 18K09091), Health Labor Sciences Research Grant, JOA-Subsidized Science Project Research from the Japanese Orthopaedic Association, JST COI grant (No. JPMJCE1302), and a grant from the Japan Orthopaedics and Traumatology Research Foundation (No. 421).

\section{Competing interests}

The authors declare no competing interests. 


\section{Additional information}

Correspondence and requests for materials should be addressed to S.O.

Reprints and permissions information is available at www.nature.com/reprints.

Publisher's note Springer Nature remains neutral with regard to jurisdictional claims in published maps and institutional affiliations.

(c) (1) Open Access This article is licensed under a Creative Commons Attribution 4.0 International License, which permits use, sharing, adaptation, distribution and reproduction in any medium or format, as long as you give appropriate credit to the original author(s) and the source, provide a link to the Creative Commons licence, and indicate if changes were made. The images or other third party material in this article are included in the article's Creative Commons licence, unless indicated otherwise in a credit line to the material. If material is not included in the article's Creative Commons licence and your intended use is not permitted by statutory regulation or exceeds the permitted use, you will need to obtain permission directly from the copyright holder. To view a copy of this licence, visit http://creativecommons.org/licenses/by/4.0/.

(C) The Author(s) 2021 